\title{
Anorexia nervosa, liquorice and hypokalaemic myopathy
}

\author{
S. Nightingale $\dagger$ \\ B.Sc., M.R.C.P.
}

\author{
P. E. SMITH* \\ M.B. B.S.
}

\author{
D. M. Turnbull $\dagger$ \\ M.R.C.P.
}

\begin{abstract}
${ }^{*}$ Royal Victoria Infirmary, and $†$ Muscular Dystrophy Laboratories, Newcastle General Hospital, Newcastle upon Tyne
\end{abstract}

\begin{abstract}
Summary
A patient with a past history of anorexia nervosa developed a hypokalaemic myopathy following a 'flulike illness. Although she was apparently in remission from anorexia nervosa, the diet was found to be markedly abnormal with an excessive ingestion of liquorice and a low potassium salt intake. The clinical features and investigations, including muscle biopsy, are described. The patient is compared with 2 reported cases of liquorice-induced myopathy, and the relationship between anorexia nervosa, liquorice and hypokalaemic myopathy is discussed.
\end{abstract}

\section{Introduction}

Liquorice, a flavouring for confectionery and soft drinks is an extract of the plant Glycyrrhiza glabra. The active ingredient of liquorice, glycyrrhizinic acid, has a chemical structure similar to aldosterone and if taken in sufficient quantities has a mineralocorticoid effect, which can be inhibited by spironolactone (Salassa, Mattox and Rosevear, 1962). A case is now reported of hypokalaemic myopathy in a young woman in whom the association of the myopathy with anorexia nervosa and liquorice abuse was not immediately apparent.

\section{Case report}

A 25-year-old married woman was admitted with a painful proximal muscle weakness. She had been well until 3 months before admission when she had suffered for a week a 'flu-like illness with some vomiting and diarrhoea. As the illness subsided she noted pain and tenderness in her proximal muscles and a progressive weakness of her limbs though not of her face or bulbar muscles. She also had had polyuria and nocturia for one month before admission; her urine was never abnormally dark. Her general practitioner had noted hypokalaemia and had prescribed oral potassium supplements for one month. She had taken no purgatives or diuretics. She had been treated for anorexia nervosa from the age of 17 years until she was discharged from psychiatric supervision when 20 years old. Since then her weight has remained stable at $54 \mathrm{~kg}$. She admitted to eating moderate amounts of liquorice in the past but denied ingesting any recently. However she had a markedly abnormal attitude to food and her own body weight, typical of anorexia nervosa. Her family confirmed the abnormal eating pattern and estimated that she had often eaten a 2-lb confectioner's box of liquorice and $8 \mathrm{oz}$ of Pontefract cakes per week.

On admission her weight was $54.5 \mathrm{~kg}$, height 177 $\mathrm{cm}, \mathrm{BP} 110 / 70 \mathrm{mmHg}$, pulse $72 / \mathrm{min}$, temperature $37^{\circ} \mathrm{C}$. General examination was normal. She had mild tenderness of her proximal muscles especially the triceps and quadriceps and a moderate (MRC grade 4) weakness of proximal limb muscles and, to a lesser extent, distal muscles. Her limbs were generally thin but there was no focal wasting or fasciculation. Sensation was intact. Her tendon reflexes and plantar responses were normal.

Investigations on admission: $\mathrm{Hb} 11.9 \mathrm{~g} / \mathrm{dl}$, with normal red blood cell indices and ESR. Potassium $1.7 \mathrm{mmol} / \mathrm{l}$; sodium $144 \mathrm{mmol} / 1$; bicarbonate 42 $\mathrm{mmol} / \mathrm{l}$; chloride $93 \mathrm{mmol} / \mathrm{l}$. Creatine kinase activity (CK) 2000 i.u./l (normal $30-70$ i.u./l). Glucose tolerance was normal. Auto-antibody screen negative. Her ECG showed severe hypokalaemic changes. Nerve conduction studies were normal but electromyography of the right tibialis anterior and quadriceps muscles showed myopathic features. A needle muscle biopsy of the quadriceps (Fig. 1) showed moderate fibre atrophy particularly affecting type II fibres with occasional necrotic fibres undergoing phagocytosis. No vacuolar changes on light microscopy were noted. Despite a low plasma potassium her daily urine potassium excretion varied from $\mathbf{8 0}$ 


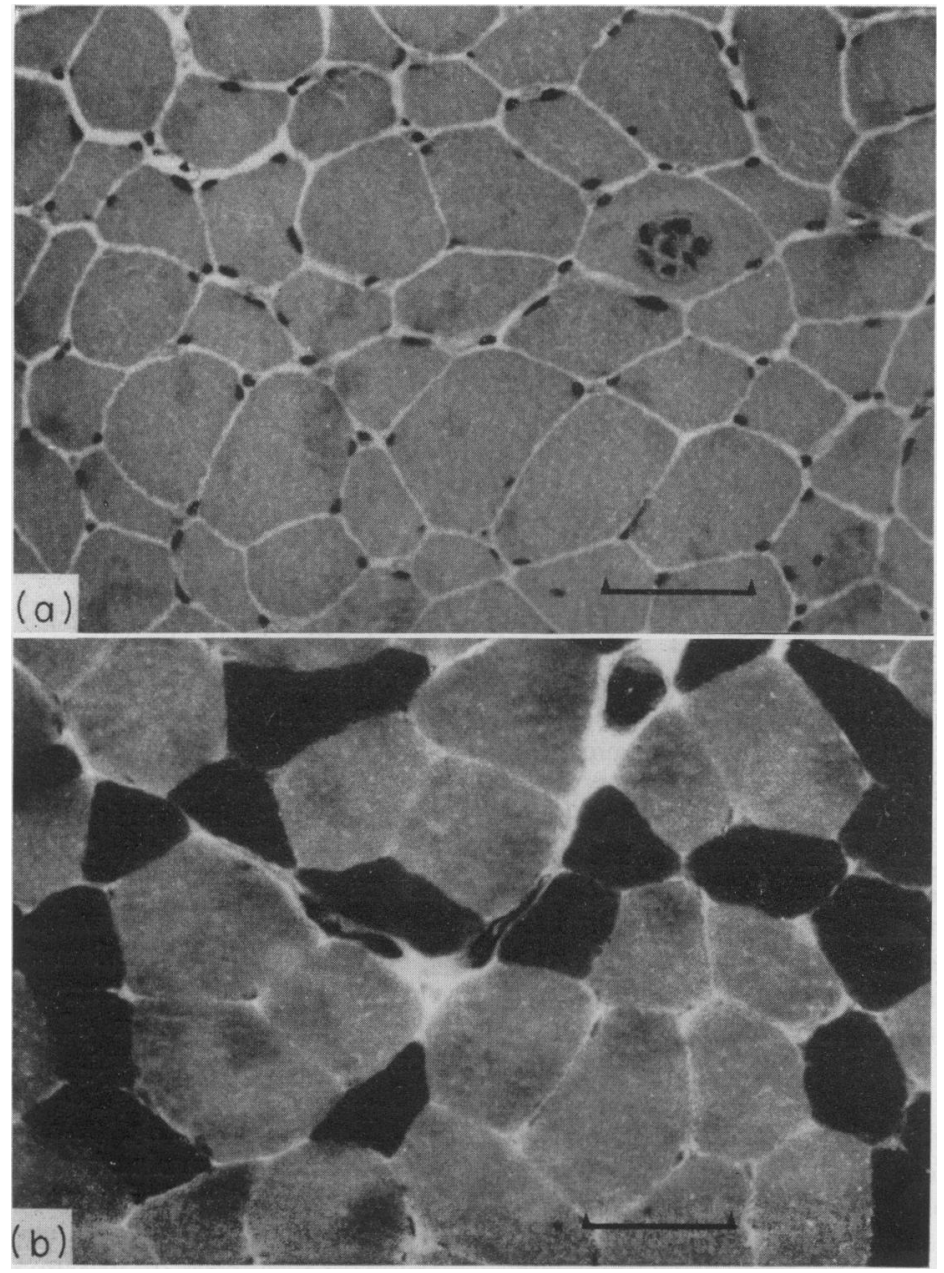

FIG. 1. Transverse section of quadriceps muscle showing (a) a necrotic fibre undergoing phagocytosis (HE); (b) moderate type II fibre atrophy (ATPase); bar represents $50 \mu \mathrm{m}$.

to $168 \mathrm{mmol}$. Urine aminoacid chromatography was normal and no myoglobin, senna or phenolphthalein were detected in her urinc. Her ability to acidify and concentrate her urine was impaired. A dietary assessment revealed a long-standing abnormally low potassium salt intake.

She was treated with oral and i.v. potassium totalling about $16 \mathrm{~g} /$ day and within 4 days her plasma potassium and ECG had returned to normal and her painful myopathy had remitted. Within one week of admission her CK was normal. Her excessive kaliuresis and renal dysfunction persisted for 3 weeks. As her myopathic symptoms abated, the patient exhibited overtly anorectic features and her weight fell. She is currently (1981) under treatment for anorexia nervosa.

\section{Discussion}

Hypokalaemic myopathy was precipitated by $\mathscr{O}_{3}$ febrile illness associated with diarrhoea and vomiting in a patient with a long history of abnormal potassium balance due to low potassium intake and excessive liquorice ingestion. With oral and pare teral potassium replacement, the plasma potassiurf, renal function, ECG, CK and myopathic sighs returned to normal.

Two patients with hypokalaemic myopathy anf excessive ingestion of liquorice have been describeg, although both were taking diuretics which woufd contribute to the hypokalaemia (Gross, Dexter angl Roth, 1966; Tourtellotte and Hirst, 1970). Both patients were obese and, like the present patien?, ate liquorice in order to lose weight. Electrolyd 
disturbance will occur with a daily ingestion of $4 \mathrm{~g}$ of glycyrrhizinic acid, the active ingredient in liquorice (Louis and Conn, 1956; Molhuysen et al., 1950); however, some patients may be particularly sensitive to the hypokalaemic effects of liquorice (Cumming et al., 1980). The patient often consumed 4 to 5 times this amount. Myoglobinuria and a severe vacuolar myopathy have been described in hypokalaemic myopathy (Gross et al., 1966; Tourtellotte and Hirst, 1970). The present patient showed neither, possibly owing to the partial potassium replacement before admission.

It is of interest that, although the patient had maintained a stable and satisfactory weight for several years and was apparently in remission from anorexia nervosa, she still indulged in abnormal eating habits, exhibited a pathological attitude to her food and was deceitful with the ward staff. Abuse of purgatives and diuretics by anorectics may also cause hypokalaemia (Nardone, McDonald and Girard, 1978) although this was not a contributing factor in the present patient. Patients with a history of anorexia nervosa who develop unusual medical problems should arouse suspicion and when possible an independent account of the patient's eating patterns should be obtained.

\section{Acknowledgments}

We thank Dr J. Anderson for permission to report his patient and Dr M. A. Johnson for reporting the muscle biopsy.

\section{References}

Cumming, A.M.M., Boddy, K., Brown, J.J., Fraser, R., LeVer, A.F., PAdfield, P.L. \& Robertson, J.I.S. (1980) Severe hypokalaemia with paralysis induced by small doses of liquorice. Postgraduate Medical Journal, 56, 526.

Gross, E.G., Dexter, J.D. \& Roth, R.G. (1966) Hypokalemic myopathy with myoglobinuria associated with licorice ingestion. New England Journal of Medicine, 274, 602.

Louis, L.H. \& ConN, J.W. (1956) Preparation of glycyrrhizinic acid, electrolyte-active principle of licorice: its effects upon metabolism and upon pituitary-adrenal function in man. Journal of Laboratory and Clinical Medicine, 47, 20.

Molhuysen, J.A., Gerbrandy, J., De Vries, L.A., De Jong, J.C., Lenstra, J.B., Turner, K.P. \& BORST, J.G.G. (1950) A liquorice extract with deoxycortone-like action. Lancet, ii, 381 .

Nardone, D.A., McDonald, W.J. \& Girard, D.E. (1978) Mechanisms in hypokalemia: clinical correlations. Medicine, 57, 435.

Salassa, R.M., Mattox, V.R. \& Rosevear, J.W. (1962) Inhibition of the 'mineralocorticoid' activity of licorice by spinonolactone. Journal of Clinical Endocrinology and Metabolism, 22, 1156.

Tourtellotte, C.R. \& Hirst, A.E. (1970) Hypokalemia, muscle weakness, and myoglobinuria due to licorice ingestion. Western Journal of Medicine, 113, 51. 